\title{
Optimiser la combustion pour un développement durable du bois-énergie
}

\author{
C. Bernard' ', M.L. Rabot-Querci' ${ }^{1}$, Y. Rogaume', \\ C. Rogaume ${ }^{1}$ et A. Zoulalian' \\ 1 Lermab, UMR Inra - Engref - UHP, \\ 27, rue du Merle Blanc, BP 1041, 88051 Epinal Cedex - France \\ e-mail : claude.bernard@enstib.uhp-nancy.fr - marie-laure.rabot-querci@enstib.uhp-nancy.fr - yann.rogaume@enstib.uhp-nancy.fr \\ caroline.rogaume@enstib.uhp-nancy.fr - andre.zoulalian@lermab.uhp-nancy.fr
}

\begin{abstract}
Résumé - Le bois-énergie, majoritairement utilisé sous forme de chauffage individuel et de chaufferies industrielles et collectives bénéficie de forts potentiels inexploités en termes de ressources et de consommateurs. C'est dans cette optique de développement que s'inscrit ce travail basé sur l'étude de 18 chaudières au bois dont la puissance varie de $20 \mathrm{~kW}$ à $4 \mathrm{MW}$ et de plusieurs appareils indépendants d'une puissance de l'ordre de $10 \mathrm{~kW}$. Le monoxyde de carbone étant un très bon indicateur de la qualité de combustion, nous avons utilisé cette mesure pour qualifier les appareils et voir l'influence de paramètres tels que la nature du foyer, le système de régulation ou la qualité du combustible sur les résultats de combustion. En effet, l'utilisation du bois comme source d'énergie ne peut être considérée comme renouvelable et participant pleinement au développement durable que si les rejets émis sont faibles et maîtrisés. A l'heure actuelle, toutes les installations satisfont aux normes de rejets en vigueur, mais il est maintenant nécessaire d'aller plus loin et d'atteindre des performances thermiques et environnementales proches de celles obtenues avec les combustibles fossiles.
\end{abstract}

\begin{abstract}
Optimization of Combustion for a Sustainable Development of Wood-Energy - Woodenergy, mainly used for private heating, industrial boilers and heating networks, includes high unexploited potentials as resources or consumers. In this perspective of development, the following work deals with the study of 18 wood fed boilers providing a power between $20 \mathrm{~kW}$ and $4 \mathrm{MW}$ and several independent heating devices providing around $10 \mathrm{~kW}$. As carbon monoxide is a very relevant indicator of the quality of combustion, we used this measurement to qualify the devices and to follow the influence of parameters such as the shape of the fireplace, the system of regulation or the quality of the fuel on the results of combustion. Indeed, the use of wood as a source of energy is considered as renewable and interesting for sustainable development only if the pollutant emissions are low and under control. Nowadays, all installations respect and pass the norms of restriction for pollutant emissions, but it is now necessary to carry on with improving in order to reach the same thermic and environmental performances as those using fossil fuels.
\end{abstract}




\section{INTRODUCTION}

Le bois est le premier matériau combustible dont l'homme s'est servi. Aujourd'hui plus que jamais le bois est une source d'énergie importante en quantité, et intéressante autant environnementalement qu'économiquement. Les quantités de bois disponibles sont très fortes, que ce soit en récupérant les sous-produits de l'exploitation du bois ou en utilisant la ressource importante qui n'est pas valorisée actuellement. Quelques filières absorbent une partie des déchets de bois qui sont transformés en panneaux de particules ou en pâte à papier, mais large partie (environ $50 \%$ ) n'est pas utilisée et reste disponible pour une valorisation énergétique [1-3].

Le bois est un combustible très utilisé (environ 9,8 Mtep en France) qui est le principal combustible renouvelable qui évite l'effet de serre contrairement aux combustibles fossiles, gaz ou fioul. Economiquement parlant, le bois exploitable en chaufferies automatiques coûte entre 0 et $0,02 €$ du kWh tandis que les énergies fossiles valent environ $0,04 €$ et l'énergie électrique $0,06 €$. Cet écart de prix sur l'énergie permet de rapidement rentabiliser une installation de chauffage automatique au bois qui reste beaucoup plus chère qu'une installation conçue pour une énergie fossile. Une autre solution valable économiquement est d'installer des chauffages d'appoint utilisant du bois bûches, permettant ainsi de diminuer en partie la consommation d'énergie fossile ou électrique.

À l'heure actuelle, la part des énergies renouvelables dans la production d'énergie primaire de l'Union Européenne correspond à $11,7 \%$, le bois-énergie représentant $54 \%$ de celles-ci. La France fournit $21 \%$ de la production d'énergies renouvelables européennes bien que le bois ne représente que $4 \%$ de la consommation totale d'énergie primaire sur le sol national [4]. Ainsi, la ressource potentielle en bois-énergie aspire à être davantage exploitée dans les domaines du chauffage individuel et des chaufferies industrielles et collectives.

Dans cette perspective de développement du bois-énergie, cette étude s'attache à mieux comprendre les principes fondamentaux d'une bonne combustion et à caractériser les principaux coproduits de la combustion, et ce pour chacune des solutions techniques existantes.

\section{Appareils indépendants}

Avec plus de $90 \%$ du nombre d'appareils en fonctionnement et plus de $85 \%$ de la consommation de bois en France, les appareils indépendants que sont les poêles, les inserts, les foyers fermés et les cheminées ouvertes génèrent la principale source d'énergie thermique produite à partir du bois Seulement, ces appareils sont régulièrement mal adaptés ou mal exploités et peuvent alors devenir générateurs d'imbrûlés tels que du monoxyde de carbone, des goudrons ou des particules solides.
Pour analyser la qualité de combustion de ce type d'appareils, nous avons réalisé des mesures sur deux appareils différents : un poêle et un foyer fermé. Des mesures d'émissions de monoxyde de carbone et de particules solides en fonction des paramètres de combustion permettent de déterminer les quantités et qualité d'émissions d'imbrûlés dans ces systèmes. Ainsi, notre approche quant à la réduction des imbrûlés se déroule en une première étape de réglage des paramètres de combustion qui permet de limiter la production, puis une seconde étape qui consiste à développer un système de filtration et de destruction de ces imbrûlés. Cette dernière démarche fait l'objet d'une étude dans le cadre d'une thèse doctorat.

\section{Chaudières automatiques}

Contrairement aux appareils indépendants, les chaudières automatiques ont un taux de pénétration du marché beaucoup plus faible mais en forte croissance. Ce développement est le résultat d'une évolution de la technologie d'automatisation qui permet d'obtenir de ces installations consommant du bois une autonomie de fonctionnement et une facilité d'utilisation identique aux installations de chauffage consommant du fioul, du gaz ou de l'électricité.

Ces chaudières utilisent un combustible formé de bois fragmenté qui permet d'alimenter mécaniquement le feu. Ainsi, l'utilisateur n'intervient qu'une à deux fois par mois pour en extraire les cendres. Pour répondre aux attentes, ce type d'installations doit également pouvoir répondre aux variations des besoins en chaleur au cours de la période de chauffage et même durant une journée. Pour maintenir une combustion optimale, il sera alors nécessaire de contrôler l'équilibre entre les débits d'air et de bois et d'utiliser la technologie de chaudière la plus adaptée au combustible choisi.

Nous avons effectué des essais sur 18 chaudières au bois d'utilisations domestiques, collectives ou industrielles dont la puissance varie de $20 \mathrm{~kW}$ à $4 \mathrm{MW}$. Ces installations ont fait l'objet de mesures d'émissions de monoxyde de carbone, de monoxyde d'azote et de poussières, pour différentes qualités du combustible, formes du foyer et techniques de régulation.

\section{PROBLÉMATIQUE ET RÉSULTATS}

La finalité d'une installation de chauffage est de produire de la chaleur. Dans le cas particulier du chauffage au bois, la source de chaleur est la réaction exothermique liée à la combustion du bois. Cette réaction est optimale selon les trois paramètres clés qui sont l'équilibre chimique, la température et le temps de séjour. L'équilibre chimique impose un rapport entre le débit de combustible et le débit d'air. Pour un dispositif donné, la température est dictée par le pouvoir calorifique du combustible, les différents débits, la capacité calorifique des gaz et les pertes thermiques dans le foyer. Le 
volume de la chambre de combustion et la température des gaz dans le foyer étant fixés, le temps de séjour des gaz est connu et peut être contrôlé en modifiant les conditions d'entrée (air et combustible). Cet enchaînement logique permet de régler les paramètres de combustion pour un matériel et un combustible choisis. Il sera aussi nécessaire dans le cas de ces installations automatiques de tenir compte des possibilités de variation de qualité du combustible en fonction des livraisons (principalement en ce qui concerne l'humidité).

\section{Le combustible}

Les appareils indépendants utilisent du bois sous forme de bûches dont le seul paramètre variable est l'humidité. Pour les chaudières automatiques, plusieurs paramètres peuvent fluctuer, comme la granulométrie, la teneur en cendres, l'humidité, etc. L'essence de bois qui peut apparaître comme une caractéristique influente en première approche est très peu importante pour la combustion, d'autant que la composition chimique élémentaire des différentes essences est stable : la base de données « BIOBIB » [5] qui regroupe un nombre conséquent de chiffres afférents à cette caractérisation montre le peu de variation.

Ainsi, la composition chimique élémentaire d'un bois type peut être exprimée sous la forme générale $\mathrm{CH}_{1,44} \mathrm{O}_{0,66}$ [6]. Le taux de cendres moyen des bois utilisés comme combustible est de $0,9 \%$ et le Pouvoir Calorifique Supérieur (PCS) est de $20030 \mathrm{~kJ} \mathrm{~kg}^{-1} \pm 3 \%$. La masse volumique apparente de bois sec varie en fonction du conditionnement du combustible : de $620 \mathrm{~kg} \cdot \mathrm{m}^{-3} \pm 1,8 \%$ pour les granulés de bois, elle est de l'ordre de $500 \mathrm{~kg} \mathrm{~m}^{-3}$ pour des bûches et peut descendre jusqu'à $144 \mathrm{~kg} \cdot \mathrm{m}^{-3} \pm 68 \%$ pour un ensemble sans distinction possible de copeaux d'usinage, plaquettes forestières, sciures, etc. [7]. Les deux autres caractéristiques essentielles de la combustion du bois fragmenté sont la granulométrie et l'humidité. Ces deux caractéristiques ont une forte influence sur le mode de combustion des particules de bois : la taille de la particule détermine son temps d'existence à l'état solide dans le foyer, et l'eau contenue dans la particule modifie l'équilibre thermochimique au sein du foyer.

La taille des particules est un paramètre qui peut fortement varier en fonction de la méthode d'obtention du bois fragmenté. Ainsi, d'après la norme autrichienne spécifique au bois énergie [8], le combustible ne doit pas contenir plus de $4 \%$ de particules passant au travers d'un crible de diamètre d'ouverture de $1 \mathrm{~mm}$ (pourcentage massique). Ce premier point s'explique par le fait que les fines particules risquent de s'envoler dans les flux d'air et de fumées. Ce phénomène implique un temps de séjour dans le foyer trop court pour que ces particules puissent brûler complètement. Dans l'autre sens, lorsque la taille de la particule augmente, son temps de montée en température croît et la vitesse de libération des gaz du lit solide varie. Or, ce temps est déterminant pour les mécanismes thermochimiques qui sont plus ou moins propices à la formation de braises et donc de combustion hétérogène sur le charbon. Ce type de combustion étant la principale source de monoxyde de carbone dans la combustion du bois [9], il est probable que la combustion de grosses particules de bois soumises à une température de foyer basse soit potentiellement plus génératrice de $\mathrm{CO}$ que celle de particules de tailles moyennes. Il faut également ajouter que la présence de particules de dimensions inadaptées au mécanisme de convoyage crée des blocages du système.

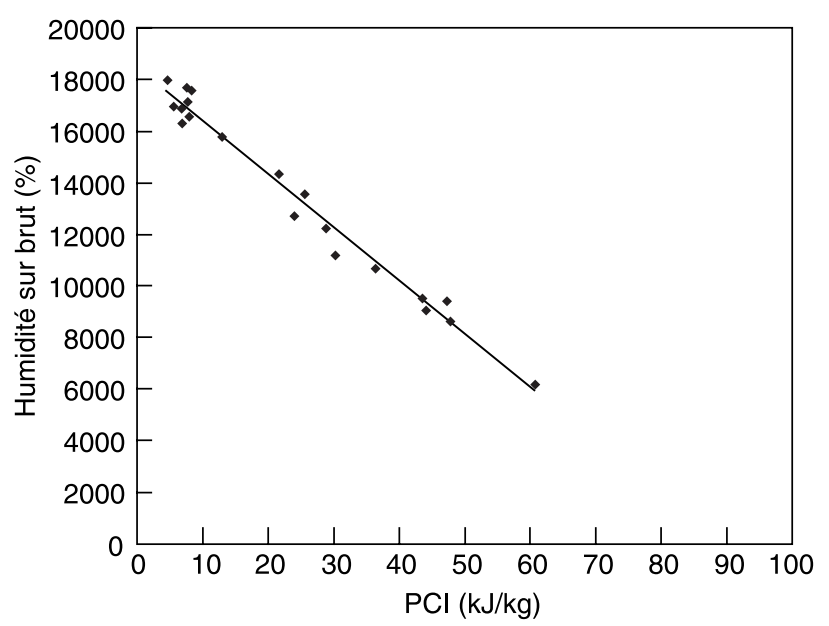

Figure 1

Pouvoir calorifique inférieur en fonction de l'humidité sur brut.

Low heating value vs. moisture content (wet basis).

La proportion d'eau contenue dans le combustible bois varie très fortement : plus de $65 \%$ de sa masse totale dans le cas d'un bois vert, entre $25 \%$ et $35 \%$ pour un bois ressuyé, $10 \%$ pour un bois séché mécaniquement et en équilibre hydrique, et jusqu'à $6 \%$ dans le cas particulier des granulés de bois. Ces quelques chiffres donnent un aperçu de la diversité de l'humidité du bois, qui entraînera des variations dans les équilibres thermiques et gazeux de la combustion. De plus, le combustible obtenu par broyage de bois vert a la faculté de perdre son eau assez rapidement lorsqu'il est entassé. Ainsi, après quelques semaines de stockage sous abri, ce bois peut atteindre une humidité sur brut de l'ordre de 35 à $40 \%$. Les silos de stockage du bois ayant des capacités plus ou moins importantes, il n'est pas rare qu'une même livraison de combustible permette de brûler au début un bois à $40 \%$ d'humidité et à la fin, un bois ayant une humidité de l'ordre de 20 ou $25 \%$. Il est alors nécessaire que la chaudière soit munie d'une technologie de régulation adaptée qui permette de contrôler la variation des flux de bois et d'air afin de maintenir le rendement thermique à son meilleur niveau. Le principal effet de l'humidité est de modifier le pouvoir 
calorifique inférieur (PCI) : celui-ci diminue fortement avec l'humidité comme le montre la figure 1 qui présente nos résultats sur 20 mesures réalisées sur des combustibles bois prélevés en chaufferies.

Il apparaît ainsi que pour obtenir le même flux thermique, le débit massique d'un bois humide doit être augmenté. Cette variation de débit massique entraîne à son tour des modifications de températures, temps de séjour, vitesses des gaz, pertes thermiques dans les fumées, cinétiques chimiques par modifications des mélanges gazeux, etc.

\section{Les appareils indépendants}

Deux appareils indépendants de taille similaire et de technologies différentes ont été testés dans des conditions de combustion de bûches spécifiées dans les normes EN 13-229 et EN 13-240. Les principales caractéristiques des appareils et du bois considéré sont regroupées dans les tableaux 1 et 2 .

TABLEAU 1

Caractéristiques des appareils

Characteristics of the devices

\begin{tabular}{l|c|c}
\cline { 2 - 3 } & Poêle & Foyer \\
\hline Puissance & $10 \mathrm{~kW}$ & $13 \mathrm{~kW}$ \\
Combustible & Bois bûches $50 \mathrm{~cm}$ & Bois bûches $50 \mathrm{~cm}$ \\
Dimension extérieure & $760 \times 738 \times 518 \mathrm{~mm}$ & $740 \times 740 \times 433 \mathrm{~mm}$ \\
Chambres de combustion & 61,51 & 931 \\
\hline
\end{tabular}

TABLEAU 2

Caractéristiques du bois

Characteristics of the wood used

\begin{tabular}{l|c}
\hline Bois & chêne \\
Longeur & $45 \mathrm{~cm}$ \\
Diamètre & $10 \mathrm{~cm}$ \\
Humidité (sur brut) & $24,30 \%$ \\
PCI $_{\mathrm{h}}$ & $15800 \mathrm{~kJ} / \mathrm{kg}$ \\
\hline
\end{tabular}

Pour étudier l'impact des diverses utilisations possibles de ces appareils, nous avons également fait varier les principaux paramètres (taux de chargement, ouvertures des entrées d'air, durée de combustion) et mesuré les émissions polluantes dans les fumées. L'impact des différents paramètres s'est révélé assez faible et difficile à déterminer d'une part, et d'autre part, nous n'avons pas pu remarquer de différences significatives entre les deux types d'appareils. Ainsi, nous présentons dans la figure 2 un exemple de résultats obtenus pour les émissions de $\mathrm{CO}$ (valeurs corrigées pour une teneur en oxygène de $13 \%$ ) pour un cycle de combustion de 2 h 30 .

Il apparait clairement sur cette courbe-type quatre phases de fonctionnement de l'appareil. Dans un premier temps

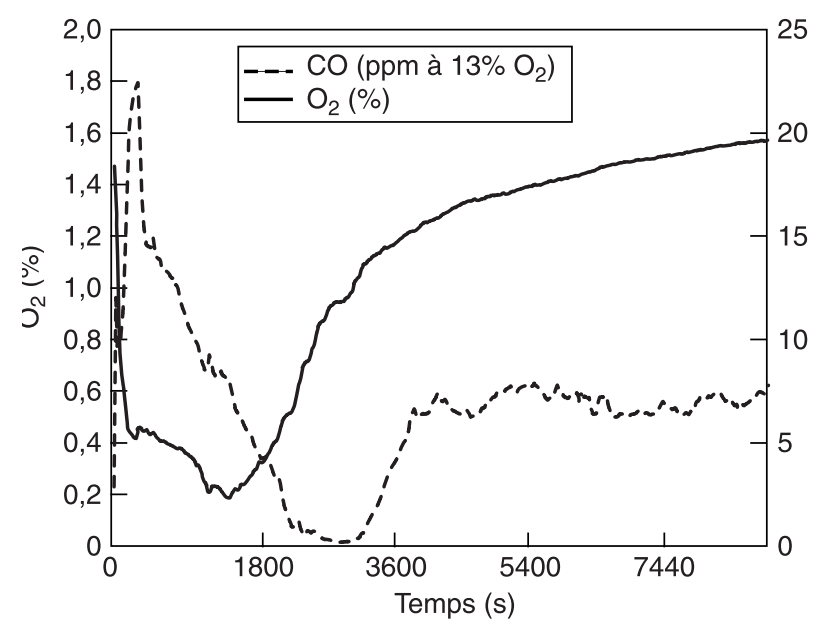

Figure 2

Emissions de CO d'un poêle au cours du temps.

Emissions of CO vs. time for a stove.

(pendant environ 10 minutes), suite au chargement, le bois chauffe lentement, dégageant de plus en plus de gaz volatils qui ont du mal à s'enflammer car la température est un peu faible et le mélange oxygène/gaz volatils relativement mauvais : durant cette phase, les émissions de $\mathrm{CO}$ sont très élevées (jusqu'à près de $2 \%$ ) et la teneur en oxygène chute. Dès lors que l'inflammation est totale, la teneur en oxygène reste faible et constante durant toute la période de combustion très vive qui dure pendant environ 30 minutes. Dans cette phase, la teneur en oxygène est inférieure à $5 \%$ et les émissions de $\mathrm{CO}$ baissent lentement jusqu'à $0,01 \%$. Ensuite, une troisième phase apparaît durant laquelle la teneur en oxygène augmente lentement, mais les émissions de CO demeurent faibles et stables : cette période dure environ 20 minutes. Enfin, la dernière phase est plus longue (1 h 30) : elle correspond à la fin du cycle, la teneur en oxygène augmente lentement entre 15 et $20 \%$ et les émissions de CO demeurent stables à un niveau voisin de $0,6 \%$.

$\mathrm{Au}$ cours des trois premières phases, la combustion se déroule majoritairement dans une phase homogène : sous l'effet de la chaleur, des gaz sont libérés par le bois, puis ces gaz, au contact de l'oxygène apporté par l'air (primaire et secondaire), sont oxydés, ce qui libère de la chaleur [10]. Au fur et à mesure de l'évolution de l'essai, une phase hétérogène apparaît, cette phase représente la combustion du charbon formé par la décomposition du bois : ce sont les braises rougeoyantes que nous voyons à la base du lit de combustible. Dans la quatrième phase de l'essai, cette combustion hétérogène devient importante et conduit à la production d'une quantité plus élevée de monoxyde de carbone.

Sur l'ensemble du cycle, les émissions de CO demeurent relativement faibles, la moyenne étant de 0,55\%. Ces mesures sont semblables à celles relevées dans la littérature 
[11-13], qui oscillent entre 0,05 et plus de $1 \%$ en moyenne sur une période d'essais dans les conditions des normes européennes. Les mêmes auteurs montrent que le rapport entre le taux de $\mathrm{CO}$ et les émissions de particules solides imbrûlées est très stable, la moyenne étant de 7,26 pour les différents types d'appareils étudiés (des cheminées ouvertes aux poêles ou foyers fermés).

Lors de nos essais, les particules solides émises lors des différentes phases ont été récoltées sur des filtres adaptés, puis analysées, notamment par microscope électronique à balayage (MEB) pour vérifier leur aspect et envisager des solutions de traitement adaptées. Cette technique permet de constater que les résidus solides sont des agglomérats d'éléments plus petits, qui s'agglutinent sans doute lors du refroidissement des gaz de combustion. Différentes études montrent que la majorité des imbrûlés solides sont des particules de diamètre inférieur à $0,9 \mathrm{~mm}$ qui représente entre 80 et $95 \%$ de la masse de poussières. Ces particules sont ultramicroscopiques avec des diamètres qui varient entre 0,05 et $0,2 \mu \mathrm{m}[14,15]$.

Il faut également noter dans les particules solides (photo du centre) récoltées la présence de nombreux éléments de bois qui ne sont pas complètement dégradés, notamment des parties de parois. En effet, les particules issues de la combustion sont très majoritairement formées par une combustion incomplète (plus de $90 \%$ [16]), qui peut même dans certains cas ne pas avoir eu lieu. Les composés formés sont ainsi de compositions différentes, plus ou moins riches en carbone, la part de matières minérales augmentant lorsque la combustion devient plus complète.

\section{Systèmes automatiques}

Dans ces systèmes, le contrôle de combustion est plus simple : le combustible bois est sous forme divisée et il est introduit dans le foyer de la chaudière au fur et à mesure des besoins de chaleur. Ainsi, la combustion est beaucoup plus homogène et il n'est plus possible de distinguer les différentes phases vues dans le cas des appareils indépendants. Les différences ou les améliorations à apporter aux systèmes sont alors la qualité du combustible ou les réglages (principalement les débits d'air).

Concernant le combustible, les deux critères déterminants sont la granulométrie et l'humidité : la taille des particules doit être en adéquation avec la technologie de transport du combustible mais par contre, nos essais ont montré que ce paramètre n'influence pas la qualité de combustion, qui demeure tout à fait semblable ; en revanche l'humidité du combustible utilisé doit être en adéquation avec la technologie du foyer de la chaudière et le principe ou les paramètres de régulation mis en œuvre [17].

La figure 4 présente les deux technologies de foyer les plus usités : un foyer à grilles et un foyer volcan. La technologie de foyer à grilles est particulièrement adaptée aux combustibles humides voire très humides car elle permet l'étalement du combustible qui doit commencer par sécher avant de brûler. Très souvent, ces chaudières munies de foyer à grilles mobiles sont équipées d'un automate de régulation qui gère la combustion en fonction de plusieurs paramètres tels que les besoins en chaleur, le taux d'oxygène dans les fumées et la pression dans le foyer. Cet équipement, au demeurant coûteux, se justifie de deux manières : le combustible peut avoir une humidité très variable et la gestion de la chaudière doit pouvoir s'adapter à cette modification automatiquement ; la technologie nécessaire et l'encombrement résultant de l'utilisation d'un combustible humide font que les foyers à grilles mobiles développent rarement moins de $250 \mathrm{~kW}$, matériel pour lequel l'ajout d'un automate de gestion ne modifie que peu le prix global d'installation. D'autres part, les combustibles humides sont souvent bien moins chers que les combustibles secs, leur utilisation étant ainsi liée surtout à un calcul économique.
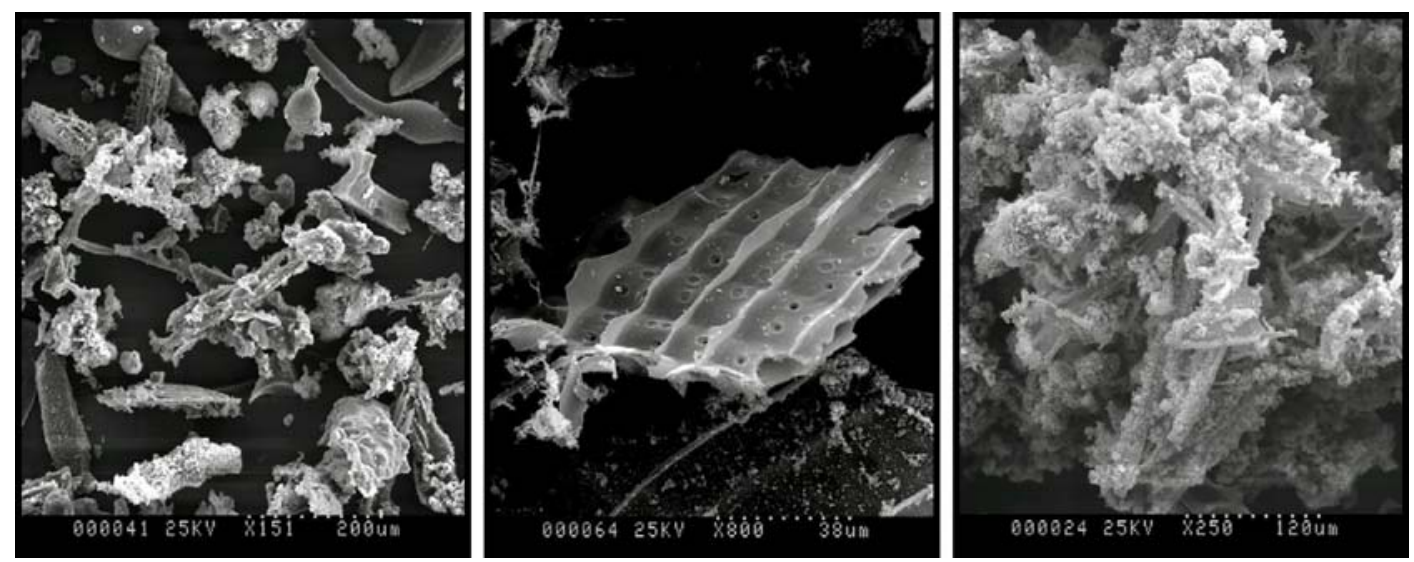

Figure 3

Vues de particules solides récoltées dans les fumées.

Views of solid particle matter collected in fumes. 


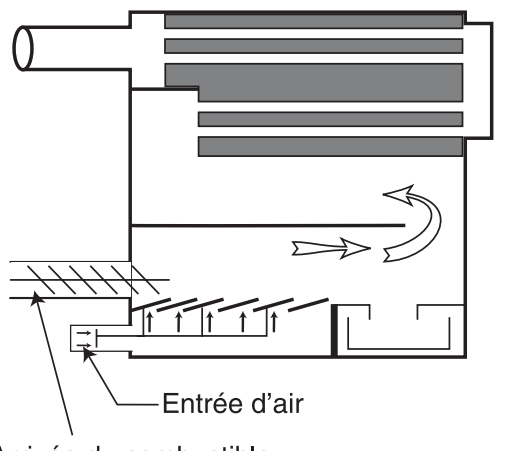

Arrivée du combustible par une vis sans fin

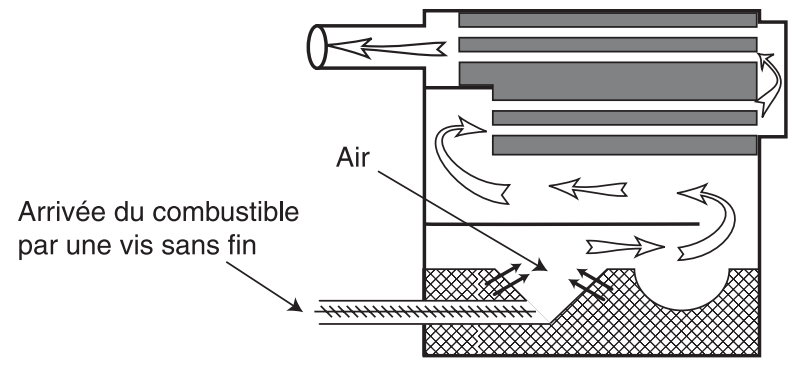

Figure 4

Vues en coupe d'un foyer à grilles (à gauche) et d'un foyer volcan (à droite).

Section of a fireplace with grates (on the left) and section of a "volcano » fireplace (on the right).
Le foyer volcan permet d'obtenir une meilleure combustion des combustibles secs tout en limitant l'encombrement du foyer. Dans cette technologie, le déplacement du combustible est vertical, et non horizontal comme dans le foyer à grilles, ce qui permet de mieux localiser les zones de réactions dans le foyer et ainsi de mieux maîtriser les temps de transit des gaz. De ce fait, la combustion est mieux contrôlée et les rendements obtenus peuvent être optimisés tout en limitant les émissions polluantes. Concernant la régulation de combustion, celle-ci peut être plus simple lorsque le combustible employé est sec et que la chaudière est de petite ou moyenne puissance (jusqu'à $100 \mathrm{~kW}$ ). La régulation se borne à mettre en route et arrêter l'alimentation en bois et en air de la chaudière en fonction de la température du fluide caloporteur contenu dans l'échangeur. Ce type de gestion n'a besoin que d'une seule information et ne délivre qu'un signal logique de type marche-arrêt (M/A), ce qui permet de limiter fortement le coût du dispositif de régulation.

\section{Répartition des technologies}

Les détails concernant la puissance, la géométrie et le principe de régulation des 18 chaudières étudiées figurent dans le tableau 3. Leurs puissances s'étendent de $20 \mathrm{~kW}$ à 4 MW. Les foyers sont de trois types : foyers à grilles fixes ou mobiles, foyers volcans (fig. 4) et brûleurs à granulés.

\section{TABLEAU 3}

Récapitulatif des 18 chaudières visitées triées par ordre décroissant d'émissions de CO Summary of 18 fed boilers graded in decreasing order of CO emissions

\begin{tabular}{|c|c|c|c|c|c|c|c|c|c|c|c|}
\hline \multirow[b]{2}{*}{ Puis. } & \multirow[b]{2}{*}{ Foyer } & \multicolumn{3}{|c|}{ Régulation } & \multicolumn{2}{|c|}{ Combustible } & \multicolumn{2}{|c|}{ Mesures } & \multicolumn{2}{|c|}{ en mg. $\mathrm{Nm}^{-3}$} & à $10 \% \mathrm{~d}^{\prime} \mathrm{O}_{2}$ \\
\hline & & $\begin{array}{r}\text { mesure } \\
\mathrm{O}_{2}\end{array}$ & $\begin{array}{r}\text { pression } \\
\text { foyer }\end{array}$ & action & $\begin{array}{l}\text { humidité } \\
\text { sur brut }\end{array}$ & $\begin{array}{l}\text { PCI en } \\
\mathrm{kJ} \cdot \mathrm{kg}^{-1}\end{array}$ & $\begin{array}{c}\mathrm{T}^{\circ} \\
\text { fumées }\end{array}$ & $\mathrm{O}_{2}$ & $\mathrm{CO}$ & NO & Poussière \\
\hline 70 & volcan & $\mathrm{N}$ & $\mathrm{N}$ & $\mathrm{M} / \mathrm{A}$ & $6 \%$ & 16400 & 189 & $12,4 \%$ & 66 & 94 & \\
\hline 20 & brûleur & $\mathrm{N}$ & $\mathrm{N}$ & $\mathrm{M} / \mathrm{A}$ & $6 \%$ & 16400 & 248 & $14,4 \%$ & 149 & 116 & \\
\hline 600 & volcan & $\mathrm{N}$ & $\mathrm{N}$ & $\mathrm{M} / \mathrm{A}$ & $10 \%$ & 14700 & 227 & $9,7 \%$ & 170 & & 89 \\
\hline 320 & grille fixe & 0 & 0 & prop. & $41 \%$ & 8500 & 181 & $11,3 \%$ & 170 & 722 & \\
\hline 350 & volcan & 0 & $\mathrm{~N}$ & prop. & $8 \%$ & 15100 & 211 & $10,7 \%$ & 173 & 475 & 110 \\
\hline 700 & grille fixe & 0 & 0 & prop. & $50 \%$ & 6900 & 270 & $2,9 \%$ & 244 & 139 & \\
\hline 55 & grille fixe & $\mathrm{N}$ & $\mathrm{N}$ & $\mathrm{M} / \mathrm{A}$ & $30 \%$ & 10800 & 230 & $12,6 \%$ & 278 & 114 & \\
\hline 4000 & grille fixe & $\mathrm{N}$ & 0 & $\mathrm{M} / \mathrm{A}$ & $6 \%$ & 15800 & 189 & $8,6 \%$ & 374 & & 189 \\
\hline 250 & grille fixe & 0 & $\mathrm{~N}$ & prop. & $39 \%$ & 8900 & 289 & $9,2 \%$ & 442 & 155 & 40 \\
\hline 1000 & grille mobile & 0 & 0 & prop. & $60 \%$ & 5400 & 238 & $6,5 \%$ & 628 & 169 & 204 \\
\hline 300 & grille fixe & $\mathrm{N}$ & $\mathrm{N}$ & $\mathrm{M} / \mathrm{A}$ & $30 \%$ & 11100 & 230 & $5,2 \%$ & 732 & 105 & \\
\hline 20 & grille fixe & $\mathrm{N}$ & $\mathrm{N}$ & $\mathrm{M} / \mathrm{A}$ & $30 \%$ & 10800 & 230 & $16,0 \%$ & 1100 & 88 & \\
\hline 55 & brûleur & $\mathrm{N}$ & $\mathrm{N}$ & $\mathrm{M} / \mathrm{A}$ & $6 \%$ & 16400 & 170 & $10,5 \%$ & 1310 & 70 & \\
\hline 30 & brûleur & $\mathrm{N}$ & $\mathrm{N}$ & $\mathrm{M} / \mathrm{A}$ & $6 \%$ & 16400 & 329 & $12,5 \%$ & 1725 & 103 & \\
\hline 40 & grille fixe & $\mathrm{N}$ & $\mathrm{N}$ & $\mathrm{M} / \mathrm{A}$ & $30 \%$ & 10800 & 439 & $8,9 \%$ & 2045 & 168 & \\
\hline 400 & volcan & $\mathrm{N}$ & $\mathrm{N}$ & prop. & $6 \%$ & 16400 & 201 & $12,6 \%$ & 2700 & 72 & \\
\hline 350 & volcan & $\mathbf{N}$ & $\mathrm{N}$ & prop. & $26 \%$ & 11300 & 297 & $9,8 \%$ & 3086 & 138 & \\
\hline 290 & grille humide & $\mathrm{N}$ & $\mathrm{N}$ & $\mathrm{M} / \mathrm{A}$ & $8 \%$ & 15300 & 230 & $6,2 \%$ & 9127 & & \\
\hline 2500 & grille mobile & 0 & $\mathrm{~N}$ & prop. & $55 \%$ & 6800 & 179 & $10,7 \%$ & 33580 & & \\
\hline
\end{tabular}


Dans le tableau 3, les installations sont triées en fonction des valeurs croissantes d'émissions de CO. Une différence est faite (séparations) en fonction du type de système de régulation mise en œuvre :

- $\mathrm{M} / \mathrm{A}$ lorsque la régulation est très simple : fonctionnement allure normale (alimentation en combustible et en air) si l'eau est sous une valeur de consigne et fonctionnement type veille (plus d'air et alimentation en combustible minimum pour entretenir des braises) lorsque l'eau a atteint la température souhaitée ;

- prop. lorsque la régulation est plus complexe : une mesure de la teneur en oxygène et de la pression sont effectuées dans le foyer. Une dépression constante est maintenue au sein du foyer, le débit de combustible et d'air primaire sont gérés proportionnellement par une boucle de régulation de type PID qui maintient toujours le système en fonctionnement à des allures variables, et le capteur d'oxygène permet de réguler le débit d'air secondaire afin de maintenir une consigne d'oxygène relativement constante dans les fumées. Il est intéressant de constater dans le tableau 3 que lorsque l'humidité du bois dépasse $30 \%$ sur brut, les systèmes de régulation mis en place sont tous de type proportionnel : cela permet d'éviter le fonctionnement de type veille qui avec du bois humide devient très polluant et peut facilement conduire à l'extinction de la chaudière.

\section{Émissions de monoxyde de carbone}

Les émissions de monoxyde de carbone étant un très bon indicateur de la qualité de combustion [18], c'est ce paramètre que nous avons choisi de suivre pour caractériser le fonctionnement des installations.

À la lecture du tableau 3, nous constatons que les trois installations les moins génératrices de $\mathrm{CO}$ sont des chaudières dénuées de régulations évoluées (notées $M / A$ ) et brûlant un combustible très sec (entre $6 \%$ et $15 \%$ d'humidité sur brut). Avec ce type de combustible, il apparaît qu'une chaudière réglée correctement permet d'obtenir une combustion optimale. Si l'on confronte ces résultats à la norme en vigueur (tableau 4), on peut voir que chacune de ces trois chaudières produisent près de 20 fois moins de $\mathrm{CO}$ que la limite supérieure bornant la classe 3 de cette norme qui est la classe des chaudières les moins polluantes.

$\mathrm{Au}$ regard de la norme, on constate également que 14 des 18 installations présentées dans le tableau 3 rentrent dans cette même classe 3 . Sur ces 14 installations, neuf sont munies d'une régulation $M / A$. Si l'on prête attention aux combustibles mis en œuvre dans ces neuf installations, il apparaît deux catégories : soit des installations brûlant un bois très sec (6-10\%) dans une technologie de foyer volcan ou brûleur, soit des foyers à grilles fixes brûlant du bois sous forme de bûches ayant une humidité de $30 \%$. La variabilité de chacun des combustibles brûlés est faible ce qui permet de bons résultats malgré la gestion simplifiée de ce type de régulations.
TABLEAU 4

Emissions seuils en CO selon la norme NF EN 303-5 Threshold emissions according to the norm EN 303-5

\begin{tabular}{c|c|c|c}
\hline \multirow{2}{*}{$\begin{array}{c}\text { Puissance utile } \\
\text { nominale }\end{array}$} & \multicolumn{3}{|c}{$\mathrm{CO}$ en $\mathrm{mg} / \mathrm{Nm}^{3}$ à $10 \% \mathrm{~d}_{2}$} \\
\cline { 2 - 4 } & classe 1 & classe 2 & classe 3 \\
\hline $\mathrm{P}<50$ & 15000 & 5000 & 3000 \\
$50<\mathrm{P}<150$ & 12500 & 4500 & 2500 \\
$150<\mathrm{P}$ & 12500 & 2000 & 1200 \\
\hline
\end{tabular}

Pour les quatre dernières lignes du tableau, il faut distinguer des fonctionnements différents :

- les deux premiers cas (chaudières de type volcan) montrent les limites de ce type d'installations : lorsque la puissance dépasse $200 \mathrm{~kW}$, la taille du volcan devient importante et il est alors difficile d'avoir un mélange homogène air/gaz combustibles au dessus du lit solide. Ce mauvais mélange mène à la formation de $\mathrm{CO}$ qu'il est ensuite difficile d'oxyder en totalité avec l'air secondaire. Dans ce cas, il semble que la séparation en deux foyers volcans placés en parallèle permettrait de mieux gérer la combustion. Il existe cependant des contre-exemples ( $3^{\mathrm{e}}$ et $5^{\mathrm{e}}$ lignes), mais il faut noter que ces deux installations brûlent des granulés de bois qui sont plus denses et qui permettent ainsi de maintenir une surface de volcan assez faible même pour des puissances élevées ;

- dans le troisième cas, les grilles utilisées sont refroidies par passage d'eau : cette technique permet d'assurer une meilleure tenue dans le temps des grilles (d'autant que le combustible est très $\mathrm{sec}$ ) et une bonne récupération de chaleur ; à l'inverse, cette technologie a tendance à refroidir davantage le foyer, ce qui peut mener à la formation d'imbrûlés de type $\mathrm{CO}$. Il convient aussi de remarquer que dans ce cas, la teneur en oxygène des fumées est relativement faible $(6,2 \%)$, ce qui peut certainement expliquer aussi en partie une production de monoxyde de carbone élevée ;

- enfin, la dernière ligne présente une installation qui est totalement déréglée et dont les émissions de CO sont extrêmement élevées. Il faut également noter que le silo de stockage et d'alimentation en combustible de la chaudière n'est pas couvert dans ce cas et qu'au moment de nos mesures, le bois était mélangé avec des quantités importantes de glace et de neige. Dans ces conditions, le système de régulation est soumis à de très fortes variations de qualité de combustible et doit réagir en permanence; le temps de réponse créant des fluctuations importantes. De plus, les éléments de glace et de neige demandent une énergie très importante pour passer de l'état solide à l'état gazeux de vapeur d'eau, ce qui abaisse la température de combustion et conduit aussi à la formation d'imbrûlés.

De manière générale, il apparaît que les chaudières ayant des dispositifs de régulation très simples de type $\mathrm{M} / \mathrm{A}$ fonctionnent très bien tant que le combustible est relativement sec 


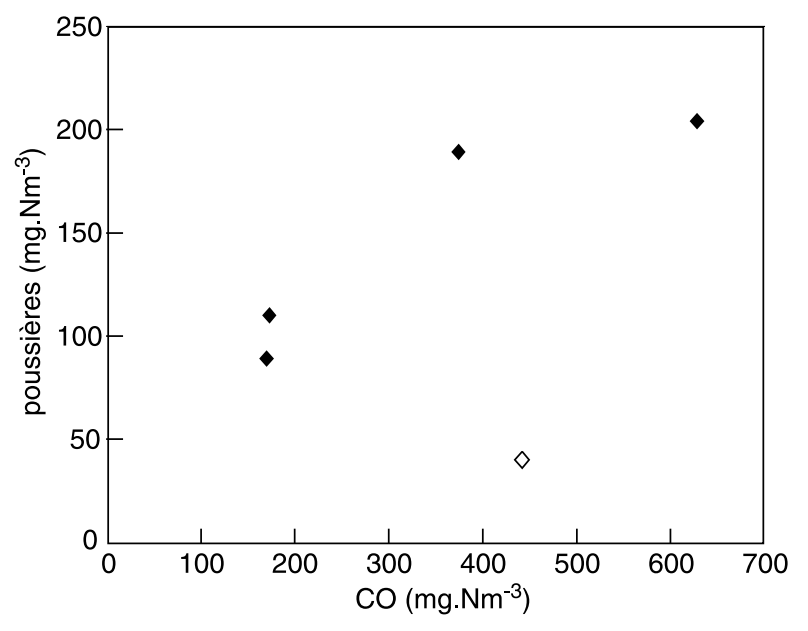

Figure 5

Corrélation entre émissions de poussières et émissions de $\mathrm{CO}$. Correlation between emissions of particle matter (PM) and CO emissions.

(humidité sur brut inférieure à $30 \%$ ) et dont les caractéristiques demeurent constantes dans le temps. Les chaudières munies d'un système de régulation proportionnel ont de très bons résultats quelles que soient les caractéristiques des combustibles utilisés et les puissances mises en œuvre. Il faut cependant noter les deux cas de régulations proportionnelles sans mesure de la teneur en oxygène : dans ces systèmes, l'automate de régulation manque de données pour ajuster au mieux les conditions de combustion. Par exemple, si l'humidité du bois augmente, le système de régulation va compenser en apportant plus de combustible, ce qui aura tendance à baisser la température au sein du foyer (entrée d'une forte quantité d'eau) ; pour compenser, la régulation va encore augmenter la quantité de combustible et le système ne pourra pas converger.

\section{Émissions de particules solides imbrûlées}

Vue la difficulté de la mesure du taux de particules dans les fumées, ces mesures n'ont pu être effectuées que sur quelques installations. En étudiant les quelques mesures dont nous disposons, nous pouvons constater une corrélation entre celles-ci et les mesures de monoxyde de carbone comme l'illustre la figure 5. Ceci s'explique simplement par le fait que monoxyde de carbone comme particules solides sont des imbrûlés issus de combustion incomplète. Ainsi, une configuration propice à la production de monoxyde de carbone, autrement dit un temps de séjour des gaz trop court, des températures trop basses, un manque d'oxygène ou autre, est également une configuration propice à la production d'imbrûlés solides.

Malgré tout, ce genre d'explication peut devenir simpliste dans notre cas car toutes les chaudières sur lesquelles les mesures de poussières ont été effectuées sont munies de dépoussiéreur cyclonique. Si bien qu'en plus de la qualité de la combustion, le taux de poussières est dépendant de l'efficacité du cyclone.

\section{Bilan}

Les résultats issus de nos mesures sur les deux grands types d'installation rencontrés dans le bois-énergie nous mène à faire un premier bilan rapide en concernant le développement durable de cette source d'énergie :

- pour les systèmes de type appareils indépendants, il apparaît que la réduction des imbrûlés à la source est difficilement compatible avec l'augmentation du rendement des appareils. Ainsi, pour continuer d'améliorer les rendements de ces dispositifs tout en limitant les émissions polluantes, il sera nécessaire de mettre en place des systèmes d'épuration des fumées ;

- pour les chaudières automatiques, il semble indispensable de choisir le générateur de chaleur en fonction du combustible disponible : il n'est par exemple pas nécessaire de choisir un automate de régulation proportionnel avec différentes sondes de mesure (oxygène, pression, etc) si le combustible demeure relativement sec et que la puissance de chaudière reste limitée ; par contre, si les caractéristiques du combustibles sont fluctuantes ou que celui-ci est assez humide, il sera indispensable de choisir une installation possédant un dispositif de régulation complet afin de garantir de bonnes conditions de combustion ;

- quel que soit le type d'installation, il apparaît clairement que les émissions de monoxyde de carbone et de particules solides par les fumées sont proportionnelles. Ainsi, le suivi des émissions de $\mathrm{CO}$ permet de qualifier la qualité de combustion et d'estimer les quantités totales d'imbrûlés. Ce résultat présent dans la littérature a aussi été confirmé par nos mesures.

À l'heure actuelle, les deux types d'appareils (appareils indépendants et chaudières) sont de plus en plus utilisés. Il convient donc de s'assurer du bon choix des systèmes et de travailler à l'amélioration des performances afin que l'utilisation du bois comme source d'énergie reste économiquement et environnementalement viable et puisse avoir toute sa place au sein des énergies renouvelables et propres.

\section{CONCLUSION}

Les normes européennes (EN 13-229, EN 13-240 et EN 303-5) mises en place entre 1999 et 2002 pour contrôler les différents appareils de chauffage au bois permettent d'améliorer les performances des divers produits. Le marquage $\mathrm{CE}$ en cours pour les mêmes appareils va encore plus loin en interdisant la production et la vente d'appareils non conformes. Ces différents points permettent ainsi un développement plus 
harmonieux du chauffage au bois qui ainsi répond aux exigences de qualité nécessaires pour assurer sa place dans le cadre du développement durable.

Il apparaît cependant que certains systèmes doivent ou peuvent être améliorés. Le premier point qui est en cours de structuration est l'approvisionnement en combustible : il est indispensable pour maintenir de bonnes performances à tous les appareils, que les combustibles (qu'ils soient sous forme de bûches, de plaquettes ou de granulés) aient des caractéristiques constantes et contrôlées dans le temps, principalement en ce qui concerne l'humidité (pour assurer une bonne combustion) ou la granulométrie (pour éviter les problèmes possibles d'acheminement du combustible dans les systèmes automatiques).

Dans un second temps, il est nécessaire que le système de combustion mis en place corresponde aux besoins (puissance adaptée) et soit choisi en fonction du combustible qui sera usité.

Pour donner suite à ces premiers résultats rappelés dans le bilan, nous menons deux études en parallèle : une étude sur la dépollution des fumées issues de combustion dans les appareils indépendants et une étude plus complète sur la combustion de bois fragmenté en chaufferies automatiques. Le premier travail consiste à mettre en place un système de filtration (de type lit solide) qui permet de piéger les particules solides. Ce filtre sera ensuite chauffé afin de dégrader les imbrûlés piégés et ainsi de ne laisser s'échapper que des fumées épurées. Ce travail en cours de développement pourra également être l'objet d'autres applications, que ce soit pour dépolluer des fumées issues de chaudières, ou de systèmes de pyrolyse ou de gazéification. Le second travail a pour objectif de déterminer plus précisément les effets de modifications de combustibles sur les émissions polluantes et le rendement d'une installation automatique. Nous avons pu voir notamment que l'humidité du combustible joue un rôle important sur l'équilibre et la qualité de combustion. Notre but est de déterminer les conditions opératoires optimales quelle que soit l'humidité, afin de mettre en œuvre une logique de régulation qui puisse permettre d'optimiser la combustion en continu malgré les variations de qualité du combustible.

\section{REFERENCES}

1 Rogaume, Y. (2005) Production de chaleur à partir de bois : Combustible et appareillage, Techniques de l'ingénieur, Paris, BE 8747.
2 Rogaume, Y. (2005) Production de chaleur à partir de bois : Installations industrielles, Techniques de l'ingénieur, Paris, BE 8748.

3 Rogaume, Y. (2005) Production de chaleur à partir de bois : Données économiques, Techniques de l'ingénieur, Paris, BE 8749.

4 Ministère de l'Economie des Finances et de l'Industrie (2002) Les chiffres clés : l'énergie, Les éditions de l'industrie, ISBN 2110939400.

5 Biobib (2004) Base de données sur les combustibles de type biomasse, http://www.vt.tuwien.ac.at/biobib/biobib.html.

6 Deglise,X. (1982) Les conversions thermochimiques du bois, Revue Forestière Française XXXIV.

7 Bernard, C. (2003), Essais de combustion de bois en foyers fermés, Journal de la SOACHIM, 16, en cours d'impression.

8 ÖNORM M 7133 (1998), 19980201 Holzhackgut für energetische Zwecke - Anforderungen und Prüfbestimmungen.

9 Skreiberg, O., Glarborg, P., Jensen, A. et Dam-Johansen, K. (1997) Kinetic NOx modelling and experimental results from single wood particule combustion, Fuel, 76, 671-682.

10 Van Loo Sjaak et Koppejan, J. (2002) Handbook of biomass combustion and co-firing, Twente University Press, The Netherlands, ISBN 9036517737.

11 US-EPA (1996) Compilation of Air Polluant Emission Factors, AP-42, Fifth Edition, Volume I : Stationary Point and Area Sources - Rapport de U.S. Environmental Protection Agency.

12 US-EPA (2001) Residential wood combustion-Revised final, US-EPA, EIIP, January 2001, 40.

13 Dasch, J.M. (1982) Particulate and gaseous emissions from wood-burning fireplaces, Environmental Science \& Technology, 16, 639-645.

14 Hueglin, C., Gaegauf, C., Künzel, S. et Burtscher, H. (1997) Characterization of wood combustion particles : Morphology, mobility and photoelectric activity, Environmental Science \& Technology, 31, 3439-3447.

15 Johansson, L.S., Tullin, C., Leckner, B. et Sjövall P. (2003) Particle emissions from biomass combustion in small combustors, Biomass \& Bioenergy, en cours d'impression.

16 Nussbaumer, Th. (2001) Relevance of aerosols for the air quality in Switzerland, Aerosols from Biomass Combustion, International Seminar IEA Bioenergy Task 32, Zürich, Switzerland, 1-9.

17 Tillman, D.A. (1978) Wood as an energy ressource, Academic Press, New York, ISBN 0126912602.

18 Bühler R. (1989) Chauffage centraux au bois : principes de base pour l'étude et l'exécution de projets, Office fédéral des questions conjoncturelles, Berne, f 724.623.

Manuscrit final reçu en février 2006

Copyright $(\mathbb{C} 2006$ Institut français du pétrole

Permission to make digital or hard copies of part or all of this work for personal or classroom use is granted without fee provided that copies are not made or distributed for profit or commercial advantage and that copies bear this notice and the full citation on the first page. Copyrights for components of this work owned by others than IFP must be honored. Abstracting with credit is permitted. To copy otherwise, to republish, to post on servers, or to redistribute to lists, requires prior specific permission and/or a fee: Request permission from Documentation, Institut français du pétrole, fax. +33147527078 , or revueogst@ifp.fr. 